\section{Prevention of Barrett's metaplasia in a human model of duodenogastro-oesophageal reflux}

\author{
Richard J. Cade, ${ }^{1,2}$ Adrian M. Fox, ${ }^{1,2}$ \\ Eamonn T. Fahy, ${ }^{1}$ Michael W. Hii ${ }^{2}$ \\ ${ }^{1}$ Hepatobiliary/Upper GI Surgery Unit, \\ Box Hill Hospital, Melbourne; \\ ${ }^{2}$ Hepatobiliary/Upper GI Surgery Unit, \\ St. Vincent's Hospital, Melbourne, \\ Australia
}

\section{Abstract}

The development of Barrett's esophagus above the anastomosis following esophagogastrectomy has been reported in several studies. In this prospective study we set out to examine the prevalence of this phenomenon in a group of patients who have been strictly adherent to post operative proton pump inhibitor (PPI) therapy. Forty-six postoesophagectomy patients were prospectively assessed by upper gastrointestinal endoscopy. Four quadrant biopsies were taken $1 \mathrm{~cm}$ proximal to the esophago-gastric anastomosis in all patients and details of endoscopic appearance, biopsy pathology, operative pathology and PPI dose were recorded. All 46 patients had been commenced on a PPI in the immediate postoperative period. Two patients were not taking a PPI regularly. The average time from operation to endoscopy was 3 years (range 0.5 to 9). Mild (Grade 1) erosive oesophagitis was observed in 5 patients. Barrett's epithelium was not identified in any patient. One patient who was taking a PPI intermittently had macroscopic columnar epithelium for $2 \mathrm{~cm}$ above the anastomosis without intestinal metaplasia. One other patient who had no macroscopic abnormality had columnar epithelium without intestinal metaplasia, seen in one of four biopsy specimens. This is the first study to assess for endoscopic evidence of neo-Barrett's following oesophagogastrectomy, where PPI therapy has been commenced in the immediate postoperative period. Columnar epithelium was present in 2 patients and intestinal metaplasia was not detected in any of the cohort. These outcomes may be due to early commencement of PPI therapy and a high level of compliance.

\section{Introduction}

Barrett's esophagus has a strong association with reflux as a causative factor. Repeated acute injury and chronic inflammation have been implicated in the development of Barrett's and its progression to malignancy. ${ }^{1}$ The patient with established Barrett's has an increased risk of cancer development. A metaanalysis by Shaheen et al. ${ }^{2}$ of 25 articles published between 1984 and 1998 concluded that the incidence of adenocarcinoma in patients with Barrett's esophagus was approximately $0.5 \%$ per patient-year with a range from $0.2 \%$ to $2.9 \%$. Barrett's mucosa development is intriguing because it seems to occur rapidly. It is unusual for the length of Barrett's to change much once discovered at endoscopy. Population studies documenting repeated endoscopies over time in a defined patient cohort have shown that Barrett's development is likely to occur suddenly and then remain quite static in its extent. Cameron and Lomboy noted that the prevalence of Barrett's increases with age, but the mean length does not, suggesting that the onset of this condition must be rapid. ${ }^{3}$ The results of successful treatment of gastroesophageal reflux (GER) on this esophageal gastric metaplasia are conflicting and it is not known whether such treatment will diminish the risk of carcinoma. ${ }^{4}$ Ideally, effective treatment of GER should be administered before the development of Barrett's mucosa.

The patient after esophagogastrectomy has had complete removal of the lower esophageal sphincter complex and this allows free reflux to wash onto the naive squamous remnant esophagus. ${ }^{5}$ After gastric tube reconstruction, the gastric tube is intrathoracic, directly attached to the remnant oesophagus. This provides a human model of gastro-duodenal reflux. The reflux has been shown to be particularly significant while in the supine position. ${ }^{4}$ The resultant reflux episodes can be asymptomatic. The tube continues to make acid and has been shown to recover its acid production capacity to almost normal over time even though mainly denervated of vagal input. ${ }^{6}$

The development of Barrett's change in the residual esophagus following esophagogastrectomy is well described. ${ }^{5,7,8}$ Unfortunately most studies have been retrospective and the reported incidence of this finding is variable from $9 \%$ to $57 \%$ (Table 1). ${ }^{4,5,7,9-13}$ A prospective study was therefore undertaken to examine the incidence of neo-Barrett's esophagus and also to consider factors that might offer an explanation for its pathogenesis.

\section{Materials and Methods}

From 1993 to 2009, 139 consecutive oesophageal resections were performed by a single surgeon (RC), an experience that has been described elsewhere. ${ }^{14}$ All operations per-
Correspondence: Eamonn T. Fahy, Boxhill Hospital, Nelson Road, Box Hill, Melbourne 3128, Australia. Tel: +61.411 .042673 .

E-mail: eamofahy@gmail.com

Key words: Barrett's esophagus, esophagectomy. proton pump inhibitors, esophageal adenocarcinoma, Barrett's metaplasia.

Contributions: RJC, study conception and design; RJC, AMF, MWH, acquisition of data; RJC, AMF, ETF, analysis and interpretation of data; RJC, AMF, MWH, drafting of manuscript; RJC, AMF, ETF, critical revision.

Conflict of interests: the authors declare no potential conflict of interest.

Received for publication: 17 April 2013.

Revision received: 30 June 2013.

Accepted for publication: 25 July 2013.

This work is licensed under a Creative Commons Attribution NonCommercial 3.0 License (CC BYNC 3.0).

(C) Copyright R.J.Cade et al., 2013

Licensee PAGEPress, Italy

Gastroenterology Insights 2013; 5:e4

doi:10.4081/gi.2013.e4

formed by that surgeon are entered into a prospectively compiled database and all patients are followed by the same surgeon. It is the surgeon's routine to perform endoscopy for symptoms as required, but also for surveillance in every patient at $1,2,3$ and 5 years post resection.

A prospective study was carried out on this patient population. We obtained ethical approval from the Eastern Health Research and Ethics Committee. Forty-six consecutive patients presenting for gastroscopy either for reflux symptoms or surveillance following esophagectomy were enrolled into the study after obtaining consent. These patients all had endoscopic assessment of the area of the anastomosis by macroscopic appearance and also 4 quadrant biopsies.

\section{Surgical procedure}

The relevant features of the esophageal resection surgery are as follows. A standard Ivor-Lewis esophagectomy is performed. The stomach is mobilized preserving the right gastro-omental and the right gastric arteries. The duodenum is Kocherized and a HeinekeMikulicz pyloroplasty performed. A gastric tube approximately $5 \mathrm{~cm}$ wide is created from a point immediately above the incisura to the greater curve at the fundus using a linear cutting stapler. Via a right postero-lateral thoracotomy the thoracic esophagus is mobilized proximally to a point that provides adequate cancer 
clearance and ensures that any Barrett's epithelium that may be present is included. The anastomosis is therefore performed at a level between the thoracic inlet and the arch of the azygos vein. It is a combined mechanical and handsewn technique with a stapled sideto-side anastomosis over $3 \mathrm{~cm}$ between the back wall of the esophagus and the gastric tube and then a 2 layered end-to-side esophagogastric anastomosis using interrupted $4 / 0$ polypropylene as previously described. ${ }^{14}$

In four patients a left rather than a right thoracotomy was performed with a similar anastomosis. Two patients had cervical anastomoses performed and both were handsewn with interrupted $4 / 0$ polypropylene.

\section{Use of medications}

PPI therapy followed a standard protocol. All patients commenced daily intravenous PPI therapy on the day of surgery. This was single dose $40 \mathrm{mg}$ Esomeprazole or equivalent PPI. When patients were able to manage oral intake postoperatively they were placed on oral formulations and advised to continue this indefinitely. PPI dose was adjusted as required to control reflux symptoms and patient compliance was discussed and reinforced at each postoperative consultation.

\section{Endoscopy}

Upper gastrointestinal endoscopy was performed by the treating surgeon. Any macroscopic evidence of reflux esophagitis or columnar epithelialization was noted and multiple biopsies taken from these areas. The presence of metaplastic columnar mucosa in the esophagus was suspected when circumferential areas or tongues of pink-appearing glandular mucosa extended from the esophagogastrostomy into the pale squamous epithelium of the cervical esophagus as previously reported. ${ }^{10}$ 4 quadrant biopsies (standard biopsy forceps) were taken $1 \mathrm{~cm}$ above the anastomosis in all patients regardless of the macroscopic appearance. The exact position of the anastomosis was evident by the persisting 4/0 polypropylene sutures (Figure 1). One of the cervical and two of the intrathoracic anastomoses required dilatations. None of these had columnar lined esophagus (CLO) above the anastomosis.

\section{Histopathologic assessment}

Specimens were submitted for histopathological assessment by experienced pathologists. All specimens underwent routine fixation and staining with haematoxylin and eosin. Columnar metaplasia was defined as the presence of esophageal columnar epithelium with or without specialized intestinal metaplasia. Barrett's epithelium was defined as the finding of specialized intestinal metaplasia with goblet cells. The histopathology of the original resection specimen was also reviewed to ensure that the resection margin was clear, in normal squamous esophageal mucosa, and therefore there was no residual Barrett's.

\section{Results}

\section{Clinical and demographic features}

46 patients ( 34 male; 12 female) with a mean age of 65 years were included in the study. The median time after esophagectomy was 3 years (range 0.5-9). Indications for the primary operation are shown in Table 2. All resected specimens had a squamous epithelial proximal margin indicating complete excision of any associated Barrett's.

\section{Use of medications}

At the time of the study 44 of the 46 patients (96\%) were taking a PPI regularly and one other intermittently. Twenty-three took a standard dose of a PPI once a day, three patients took an additional dose as needed according to symptoms and 18 patients took double dose PPI. The patient undergoing the McKeown esophagectomy was treated with PPI immediately postoperatively however discontinued the drug after discharge from hospital.

\section{Endoscopy results}

Five patients had mild (Grade 1) erosive esophagitis. One patient who was taking a PPI intermittently had macroscopic columnar epithelium for $2 \mathrm{~cm}$ above the anastomosis without intestinal metaplasia. One other patient who had no macroscopic abnormality had columnar epithelium without intestinal metaplasia, seen in one of four biopsy specimens. Barrett's esophagus was not identified in any patient.

\section{Discussion}

After Ivor-Lewis esophagectomy, normal proximal esophagus is juxtaposed to gastric mucosa without an intervening sphincter mechanism thereby allowing acid and duodenal fluid to reflux onto naive squamous mucosa of the remaining esophagus. ${ }^{15}$ As a result, symptomatic reflux is common and we routinely prescribe patients a PPI postesophagectomy.

PPI therapy was introduced progressively into clinical practice in Australia from 1989 and is subsidized by the national Pharmaceutical Benefits Scheme. ${ }^{16}$ The

Table 1. Summary of postoesophagectomy studies.

\begin{tabular}{|c|c|c|c|c|c|c|c|}
\hline Study (ref) & Year & No. Pts & $\begin{array}{c}\text { Endoscopy } \\
(\%)\end{array}$ & $\begin{array}{c}\text { Biopsy } \\
(\%)\end{array}$ & $\begin{array}{c}\% \text { CLO } \\
\text { (number) }\end{array}$ & $\begin{array}{c}\text { \% Barrett's } \\
\text { (number) }\end{array}$ & $\begin{array}{c}\text { PPI use } \\
(\%)\end{array}$ \\
\hline Lindahl (4) & 1990 & 14 & 100 & 72 & $72(10)$ & $57(8)$ & NR \\
\hline Hamilton (5) & 1977 & 17 & 10 & $17^{*}$ & $41(7)$ & $18(3)$ & 0 \\
\hline O'Riordan (7) & 2004 & 48 & 100 & 100 & $50(24)$ & $20(14)$ & 58 \\
\hline Wolfsen (9) & 2004 & 45 & 80 & 80 & NR & $22(8)$ & $\mathrm{NR}^{\circ}$ \\
\hline Oberg (10) & 2002 & 32 & 100 & 100 & $46.9(15)$ & $9(3)$ & $68.8^{\sharp}$ \\
\hline Franchimont (11) & 2003 & 66 & 100 & 100 & $9(6)$ & $13.5(9)$ & $74^{\S}$ \\
\hline Lord (12) & 2004 & 20 & 100 & 100 & $50(10)$ & $25(5)^{\wedge}$ & NR \\
\hline Dresner (13) & 2003 & 40 & 100 & $100^{\$}$ & $48(19)$ & $23(9)$ & NR \\
\hline Rice (19) & 2011 & 231 & 100 & 100 & $33(76)$ & $8.7(20)$ & 59 \\
\hline Cade & 2010 & 46 & 100 & 100 & $4(2)$ & 0 & 96 \\
\hline
\end{tabular}

CLO, columnar lined esophagus; PPI, proton pump inhibitor; NR, not reported. Biopsy was obtained from autopsy (6), endoscopic biopsy (10) or at operation (1). Twice-daily proton pump inhibitors were routinely prescribed for these patients although patient compliance is difficult to assess because of high drug costs and limited symptomatic improvement. While the small number of patients limits our analysis, these factors were found to occur in a proportional number of patients with Barrett's disease and no clear trends could be identified. "Eight of these patients were being treated with a single daily dose of proton pump inhibitors and 12 were receiving proton pump inhibitors twice daily. Two patients were being treated with $\mathrm{H} 2$ receptor antagonists. §PPI use started median 87 days after surgery in patients without BE, (range 0 -1020 days), versus 167 days with BE, (range 0-541 days); P>0.05. `One patient had developed adenocarcinoma. 'Biopsy taken $2 \mathrm{~cm}$ 's above anastomosis. 
affordable cost of these medications, together with regular review and encouragement by the treating surgeon, results in a high compliance rate (44 of 46 patients). A variety of PPIs are available (esopmeprazole, lansoprazole, omeprazole, pantoprazole and rabeprazole), however the differences between them are probably not of clinical significance. ${ }^{17}$

Although we did not observe any patient developing neo-Barrett's, this is not the experience of others (Table 1). Two patients in our study developed columnar lined lower esophagus. A recent review, based on several series, estimated that columnar lined esophagus was expected to occur in $\geq 50 \%$ of patients after esophagectomy with gastric pull-up. ${ }^{18}$

In a retrospective review of post-esophagectomy patients, Wolfsen et al. ${ }^{9}$ found Barrett's to be present in 8 out of 36 patients, all of whom were routinely prescribed twice-daily proton pump inhibitors. However, compliance was a major problem which they attributed to high drug costs and limited symptomatic improvement. ${ }^{9}$

Wolfsen's study population received esophagectomy for a variety of Barrett's related disorders. It had been thought that this group might be at particular risk of developing neo-Barrett's in the residual esophagus. ${ }^{10}$ However, other studies have shown that the development of Barrett's post esophagectomy is not limited to a previous diagnosis of adenocarcinoma or Barrett's epithelium. ${ }^{7}$ Our patient population received esophagectomy for a range of pathologies (Table 1).

Franchimont et al. ${ }^{11}$ also reported on PPI use and post-esophagectomy Barrett's development. They remarked that Prophylactic PPI treatment directly after surgery might prevent postoperative development of Barrett's esophagus. Evidence of neo-Barrett's was found in 9 out of 66 patients post-esophagectomy in their retrospective case note review. A further 6 patients had cardiac mucosal lining in the supra-anastomotic esophagus. Thus a total of 15 patients (23\%) had columnar lined esophagus. PPI use in this study was reported as $74 \%$. However, the median interval between surgery and commencing a PPI was 167 days in the patients who developed Barrett's.

In a similar study to ours from Ireland, ${ }^{7} 48$ post esophagectomy patients underwent endoscopy and biopsy. Twenty-four had developed columnar mucosa in the residual oesophagus and in 14 of these intestinal metaplasia was demonstrated. Twenty-eight patients had been taking a PPI, however it was not recorded when this had been commenced.

A recent study from the Cleveland Clinic has provided further evidence for the use of PPIs following esophagectomy. ${ }^{19}$ Anti-reflux medication, induction therapy and higher anastomosis were found to be predictive of less
Table 2. Indications for esophagectomy $(n=46)$.

$$
\text { Indication }
$$
No.

Squamous cell carcinoma

Adenocarcinoma

26

High grade dysplasia/in situ adenocarcinoma in Barrett's

6

Achalasia

Gastrointestinal stromal tumor

Crohn's disease

1

changes consistent with reflux in biopsies which were obtained a median of 3.5 years after esophagectomy. It is interesting to note that the prevalence of reflux changes remained constant at $92 \%$ throughout the 10 year study period - implying that these changes occur early. Fifty-nine percent of patients were receiving anti-reflux therapy (primarily PPIs), prescribed as needed for reflux symptoms. The interval between esophagectomy and commencing anti-reflux medication was not recorded.

The relationship between time of esophageal injury and commencement of acid suppression is probably important. In 1970 Bremner published the results of an experiment in which he removed the mucosa from the distal esophagus in 35 dogs. ${ }^{20}$ The group was divided into three according to the presence or absence of gastro-esophageal reflux and gastric hyper-secretion. They found that re-epithelialization by squamous epithelium predominated in preparations with a competent lower esophageal sphincter. On the other hand, total or nearly total replacement by columnar cells characterized those preparations with gastro-esophageal reflux and gastric hyper-secretion. Re-epithelialization of the denuded lower esophagus with a columnar lining occurred fairly quickly, not requiring long term or pulsatile acid exposure. This study was conducted in the pre PPI era. A modern human equivalent of this study would be the recent trend towards endoscopic ablation of Barrett's under the cover of PPI therapy resulting in healing with squamous epithelium. ${ }^{21}$

Other authors have found that neo-Barrett's can occur soon after esophagectomy. This phenomenon has been described at 3 months postoperatively even when all of the original Barrett's change had been carefully excised or when the original operation was not for Barrett's related disease. ${ }^{10}$ Franchimont noted its development after 43 days. ${ }^{11}$

Providing further support for the speed with which Barrett's can develop, Cameron and Lomboy showed that the prevalence of Barrett's esophagus increased with age, consistent with an acquired disorder, but that the mean length of columnar epithelium did not increase with age. They did this by studying a statewide database of all endoscopies per-

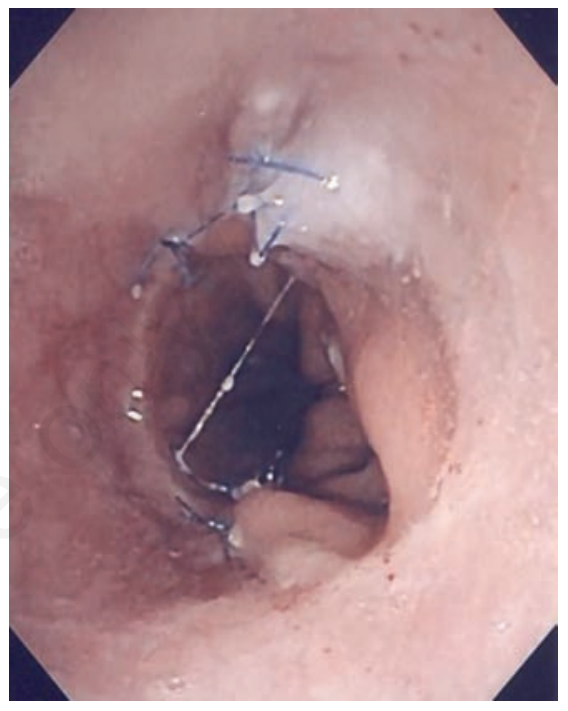

Figure 1. Endoscopic photograph showing the cervical esophagus above the gastroesophageal anastomosis, made clear by the persistent $4 / 0$ polypropylene sutures.

formed in California in the 1970's and 1980's. The authors concluded that the onset of the condition must be rapid. ${ }^{3}$

It has been suggested that PPI therapy is unnecessary because these patients have had a vagotomy. However, Gutschow in 2001 showed that the intraluminal $\mathrm{pH}$ in the gastric tube returns to normal with time after oesophagectomy. ${ }^{6}$

The role of the pyloroplasty in aggravating esophageal reflux by promoting duodenogastric reflux or reducing esophageal reflux by improving gastric drainage is debated. Oberg et al. ${ }^{10}$ and 0 'Riordan et al. ${ }^{7}$ have both found no significant difference in the prevalence of abnormal esophageal bilirubin exposure in patients with and without pyloroplasty. In any event, the carcinogenic effects of bile refluxate are dependent upon acidification with a lower $\mathrm{pH}$ required for bile salts to cross mucosal cell membranes to cause intracellular injury. ${ }^{22}$

No antireflux type anastomosis was performed in our series, but this is an alternative strategy to both control reflux symptoms and to attempt to prevent columnar epithelializa- 
tion. ${ }^{23}$ However, it would be important that after such a procedure early endoscopy was performed to ensure that reflux esophagitis was not occurring. Twelve percent of these patients may be troubled symptomatically by severe reflux. ${ }^{23}$ This rate of symptomatic reflux has been confirmed in a recent prospective trial. ${ }^{24}$ Lindahl et al. ${ }^{4}$ studied a pediatric population and reported their results in 1990 before the widespread introduction of PPI therapy. They studied children who were at least 2 years post-esophagectomy and found columnar lined esophagus in 8 of 14 patients. It was their conclusion at that time that all postesophagectomy patients be treated with histamine 2 receptor antagonists.

The main limitation of this study is that we have not randomized our patient sample to PPI use or none. This would be difficult because of the substantial symptomatic improvement with the use of a PPI that most patients with reflux benefit from. A comparison arm of antireflux anastomosis would be possible; however the recent randomised study performed did not report on endoscopic rate of Barrett's or CLO and some patients still required a PPI. ${ }^{23}$

Ambulatory 24-hour pH monitoring was not performed. We did not consider these investigations would add relevance to our study endpoints which were endoscopic appearance and histopathology of biopsy samples above the anatomosisis. Also the use of aspirin and nonsteroidal anti-inflammatory drugs (NSAIDs) were not recorded. These medications may have a minor role in chemoprevention of Barrett's development or its malignant progression, but their effect is probably small compared to the possible effect of $\mathrm{PPI}^{24}$ (and in some studies a negative effect has been shown $^{25}$ It is interesting to note recent data which suggests a lower rate of Barrett's among patients of African, Asian and Hispanic decent. ${ }^{26,27}$ Our study population was predominantly Caucasian (88\%), with lower numbers of patients of Asian (9\%), Hispanic (1.5\%) and African (1\%) backgrounds. This would suggest that race was not a significant contributor to our results.

Compliance testing was not performed to assess the true rate of PPI use. The use of pill counting, interrogation of the national PBS pharmacy register or specific structured questionnaire were not employed. The unique aspect of this population is that a single surgeon follows all patients. Detailed discussion of reflux symptoms are explored with every follow-up consultation as it is recognized by the investigators that substantial improvement in quality of life after esophagectomy can be achieved by control of reflux symptoms.

\section{Conclusions}

In this prospective study neo-Barrett's esophagus following esophagogastrectomy was not observed. This may be due to the fact that that gastric acid suppression with PPIs was commenced in the immediate postoperative period and long term compliance with medication was high. A high incidence of neoBarrett's has been reported in other studies in which PPI use was sporadic. Our recommendation is that early PPI use should be considered for all patients who undergo an esophagogastrectomy, who do not have an antireflux anastomosis. In our opinion, this anti-reflux therapy should be continued indefinitely.

\section{References}

1. Fitzgerald RC. Molecular basis of Barrett's oesophagus and oesophageal adenocarcinoma. Gut 2006;55:1810-20.

2. Shaheen NJ, Crosby MA, Bozymski EM, Sandler RS. Is there publication bias in the reporting of cancer risk in Barrett's esophagus? Gastroenterology 2000;119:333-8.

3. Cameron AJ, Lomboy CT. Barrett's esophagus: age, prevalence, and extent of columnar epithelium. Gastroenterology 1992;103:1241-5.

4. Lindahl H, Rintala R, Sariola H, Louhimo I. Cervical Barrett's esophagus: a common complication of gastric tube reconstruction. J Pediatr Surg 1990;25:446-8.

5. Hamilton SR, Yardley JH. Regnerative(sic) of cardiac type mucosa and acquisition of Barrett mucosa after esophagogastrostomy. Gastroenterology 1977;72:669-75.

6. Gutschow C, Collard JM, Romagnoli R, et al. Denervated stomach as an esophageal substitute recovers intraluminal acidity with time. Ann Surg 2001;233:509-14.

7. O'Riordan JM, Tucker ON, Byrne PJ, et al. Factors influencing the development of Barrett's epithelium in the esophageal remnant postesophagectomy. Am J Gastroenterol 2004;99:205-11.

8. da Rocha JR, Cecconello I, Zilberstein B, et al. [Barrett esophagus in the esophageal stump after subtotal esophagectomy with cervical esophagogastroplasty]. Rev Hosp Clin Fac Med Sao Paulo 1992;47:69-70. [Article in Portuguese].

9. Wolfsen HC, Hemminger LL, DeVault KR. Recurrent Barrett's esophagus and adenocarcinoma after esophagectomy. BMC Gastroenterol 2004;4:18.

10. Oberg S, Johansson J, Wenner J, Walther B. Metaplastic columnar mucosa in the cervical esophagus after esophagectomy. Ann Surg 2002;235:338-45.

11. Franchimont D, Covas A, Brasseur C, et al.
Newly developed Barrett's esophagus after subtotal esophagectomy. Endoscopy 2003;35:850-3.

12. Lord RV, Wickramasinghe $\mathrm{K}$, Johansson JJ, et al. Cardiac mucosa in the remnant esophagus after esophagectomy is an acquired epithelium with Barrett's-like features. Surgery 2004;136:633-40.

13. Dresner SM, Griffin SM, Wayman J, et al. Human model of duodenogastro-oesophageal reflux in the development of Barrett's metaplasia. Br J Surg 2003;90:1120-8.

14. Epari K, Cade R. Oesophagectomy for tumours and dysplasia of the oesophagus and gastrooesophageal junction. ANZ J Surg 2009;792517.

15. Aly A, Jamieson GG. Reflux after oesophagectomy. Br J Surg 2004;91:137-41.

16. Hillman LC, Chiragakis L, Shadbolt B, et al. Proton-pump inhibitor therapy and the development of dysplasia in patients with Barrett's oesophagus. Med J Aust 2004; 180:387-91.

17. Miner P Jr, Katz PO, Chen Y, Sostek M. Gastric acid control with esomeprazole, lansoprazole, omeprazole, pantoprazole, and rabeprazole: a five-way crossover study. Am J Gastroenterol 2003;98:2616-20.

18. Oh DS, Demeester SR. Pathophysiology and treatment of Barrett's esophagus. World J Gastroenterol 2010;16:3762-72.

19. Rice TW, Goldblum JR, Rybicki LA, et al. Fate of the esophagogastric anastomosis. J Thorac Cardiovasc Surg 2011;141:875-80.

20. Bremner CG, Lynch VP, Ellis FH Jr. Barrett's esophagus: congenital or acquired? An experimental study of esophageal mucosal regeneration in the dog. Surgery 1970;68:209-16.

21. Rees JR, Lao-Sirieix P, Wong A, Fitzgerald RC. Treatment for Barrett's oesophagus. Cochrane Database Syst Rev 2010:CD004060.

22. Schweitzer EJ, Bass BL, Batzri S, Harmon JW. Bile acid accumulation by rabbit esophageal mucosa. Dig Dis Sci 1986;31:1105-13.

23. Aly A, Jamieson GG, Watson DI, et al. An antireflux anastomosis following esophagectomy: a randomized controlled trial. J Gastrointest Surg 2010;14:470-5.

24. Nguyen DM, El-Serag HB, Henderson L, et al. Medication usage and the risk of neoplasia in patients with Barrett's esophagus. Clin Gastroenterol Hepatol 2009;7:1299-304.

25. Gatenby PA, Ramus JR, Caygill CP, et al. Aspirin is not chemoprotective for Barrett's adenocarcinoma of the oesophagus in multicentre cohort. Eur J Cancer Prev 2009;18:381-4.

26. Corley DA, Kubo A, Levin TR, et al. Race, ethnicity, sex and temporal differences in Barrett's oesophagus diagnosis: a large community based study, 1994-2006. Gut 2009;58: 182-8.

27. Abrams JA, Fields S, Lightdale CJ, Neugut AI. Racial and ethnic disparities in the prevalence of Barrett's esophagus among patients who undergo upper endoscopy. Clin Gastroenterol Hepatol 2008;6:30-4. 